\title{
Three-dimensional supernova simulation of SN 1987A pro- genitor with implications for multi-messenger signals
}

\author{
Ko Nakamura ${ }^{1,2, *}$ \\ ${ }^{1}$ Department of Applied Physics, Fukuoka University, Fukuoka 814-0180, Japan \\ ${ }^{2}$ Research Institute of Stellar Explosive Phenomena, Fukuoka University, Fukuoka 814-0180, Japan
}

\begin{abstract}
Recently a new progenitor model for SN 1987A has been constructed. This progenitor model is based on a slow-merger of $14 M_{\odot}$ and 9 $M_{\odot}$ stars and it satisfies most of the observational constraints such as red-toblue evolution, lifetime, total mass and position in the Hertzsprung-Russell diagram at collapse, and chemical anomalies. We perform a three-dimensional self-consistent core-collapse supernova simulation using this new progenitor model and find that it successfully presents an explosion and leaves a $1.53 M_{\odot}$ neutron star. Assuming a detector sensitivity of Kamiokande-II and the distance to the supernova of $51 \mathrm{kpc}$, we obtain 16 neutrino detection events in one second. Some characteristic modes in its gravitational wave signal are also discussed in this article.
\end{abstract}

\section{Introduction}

SN 1987A emerged in the Large Magellanic Cloud located at a distance of $51.4 \pm 1.2 \mathrm{kpc}$. Multiwavelength studies of SN 1987A have provided unprecedented details of supernova features such as the time evolution of the bolometric luminosity and energy spectrum at all wave bands from infrared to $\gamma$-ray. Moreover, a supernova neutrino burst was detected by several neutrino detectors such as Kamiokande-II, and Baksan. Although only about two dozen of the $\sim 10^{28}$ supernova neutrinos that passed through the Earth were detected, they provide us with the first (and ever only one) direct evidence of the supernova driven by the collapsing core of a dying star. This detection implies that there had been a proto-neutron star at least for 10 seconds and it declares the dawn of the neutrino astrophysics.

The light curve and spectra indicate that SN 1987A has roughly typical explosion energy $\left(\sim 1.2 \times 10^{51} \mathrm{erg}\right)$ and ${ }^{56} \mathrm{Ni}$ mass $\left(0.07 M_{\odot}\right)$. On the other hand, SN 1987A is a unique supernova in many senses. First, a blue hot surface of the progenitor star Sk-69 202 found in the pre-explosion images, as well as the light curve without the typical plateau phase of type II-P SNe and the relatively short period of time delay (three hours) between the neutrino burst detection and the shock breakout emission, suggest that the progenitor was a blue-supergiant (BSG). Second, this BSG star is considered that it used to be a RSG at $\sim 2 \times 10^{4} \mathrm{yr}$ since three ring-like nebulae are surrounding the supernova remnant with the high $\mathrm{He}$ the $\mathrm{CNO}$ abundance ratios and the expansion velocity comparable to the RSG wind velocity.

\footnotetext{
*e-mail: nakamurako@fukuoka-u.ac.jp
} 
To explain these anomalous features, many pre-explosion evolution scenarios of the progenitor star have been proposed soon after the emergence of SN 1987A: extreme-massloss models, helium-enrichment models, low-metallicity models, rapid-rotation models, and restricted-convection models. Also, binary interaction has been considered from early on: accretion models, companion models, and merger models. See [1] for a review of these classical progenitor models.

One of the possible scenarios to explain these features is a slow merger of binary stars where the stars in a close binary evolve into a common envelope phase then the secondary star is gradually being dissolved inside the common envelope in a much longer time scale than the dynamical time scale of the secondary. Recently a new progenitor model based on the slow merger scenario has been constructed [2]. In particular, the progenitor model successfully reproduces most of the anomalous SN 1987A features such as red-to-blue evolution, lifetime, total mass and position in the Hertzsprung-Russell diagram at collapse, and the chemical anomalies.

In this article, we report the results of our self-consistent 3D simulations for core-collapse $\mathrm{SN}$ employing this new progenitor model. We solve hydrodynamic evolution and energydependent neutrino transport from the center to the outer boundary at $10,000 \mathrm{~km}$. The hydrodynamics code that we employ in this work is essentially the same as in our previous studies $[3,4]$. For neutrino transfer calculation we use the state-of-the-art neutrino opacity [5] and solve the neutrino transport taking 20 energy bins with an upper bound of $300 \mathrm{MeV}$ for electron, anti-electron, and heavy-lepton neutrinos.

\section{Results}

We perform a 3D self-consistent core-collapse supernova simulation using the latest binary progenitor model of SN 1987A. We obtain the explosion energy of $1.5 \times 10^{50} \mathrm{erg}$ and ${ }^{56} \mathrm{Ni}$ mass of $\sim 10^{-2} M_{\odot}$ and these values are still growing at the end of the simulation.

\subsection{Shock evolution}
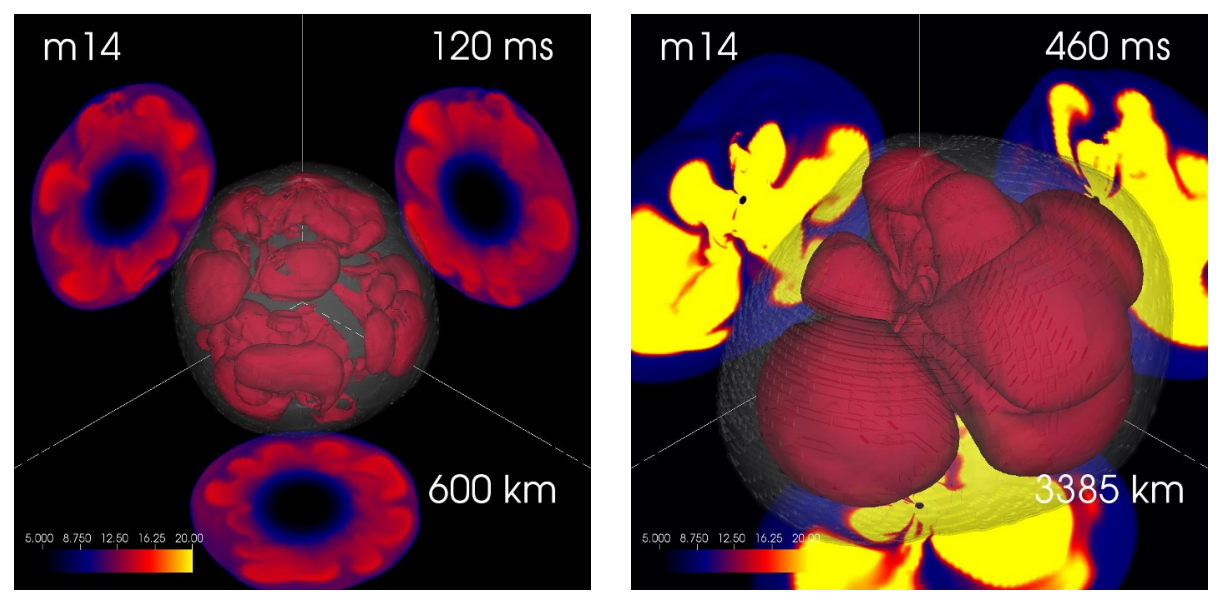

Figure 1. Entropy isosurface within the inner region of the exploding SN 1987A progenitor at $120 \mathrm{~ms}$ (left panel) and $460 \mathrm{~ms}$ (right panel) after bounce. Note the different box sizes between the panels. 
The shock of our 3D model once stalls at $r \sim 150 \mathrm{~km}$ and turns to expand at $\sim 220 \mathrm{~ms}$ after bounce. The left panel of Figure 1 shows the entropy isosurface of the inner region of our 3D model within a $600 \mathrm{~km}^{3}$ box at $120 \mathrm{~ms}$ after bounce. The matter behind the shock is heated by neutrino and small-scale convective motions are developed. The shock is finally pushed outward via the neutrino heating and at $460 \mathrm{~ms}$ after bounce it reaches $\sim 1500 \mathrm{~km}$ from the center (the right panel of Figure 1). At this later phase, the morphology of the expanding matter is dominated by a few large-scale plumes and low-entropy downflows between them. Although our simulation is limited by the outer boundary at $10,000 \mathrm{~km}$, the shock never recedes after the shock revival and it would successfully make a supernova explosion when it reaches a stellar surface.

\subsection{Neutrino signal}

Core-collapse SN emits a huge number of neutrinos. We estimate a cumulative neutrino detection event number assuming that a CCSN located at LMC emits neutrinos like our 3D CCSN model and the neutrino emission is nearly isotropic. We observe 14 neutrinos for 0.7 $\mathrm{s}$ by Kamiokande-II (K-II) and an appropriate extrapolation gives 16 events in $1 \mathrm{~s}$. These numbers are larger than, but not so far from, the detection events in the case of SN 1987A (from this historical SN, K-II observed 11 neutrinos in $\sim 10 \mathrm{~s}$ ).

\subsection{Gravitational wave signal}

Gravitational wave from coalescence of compact objects such as black holes and neutron stars has been detected by laser interferometers. Non-spherical motions of dense matter in a CCSN core also produce the gravitational wave and we expect its detection in the future. The gravitational wave from CCSN is characterized by three phases: a prompt signal soon after the core bounce followed by a silent phase, a strong signal stimulated by hydrodynamic instabilities such as convection and Standing Accretion Shock Instability (so-called SASI) lasting for a few hundred $\mathrm{ms}$, and the final explosion phase. Independent of the direction to observers, the spectrogram of our CCSN model presents that the peak frequency of the strong signal shifts from $\sim 500 \mathrm{~Hz}$ at $150 \mathrm{~ms}$ to $>1000 \mathrm{~Hz}$ at $500 \mathrm{~ms}$ after bounce. This feature is fitted by a turn-over frequency of downflows hitting the central proto-neutron star surface and reflected at a stable region against convection.

\section{Acknowledgments}

This study was supported in part by Grants-in-Aid for Scientific Research of the Japan Society for the Promotion of Science (JSPS, Nos. JP20K03939, JP21H01121), the Ministry of Education, Science and Culture of Japan (MEXT, Nos. JP20H04748, JP20H05255), by the Central Research Institute of Explosive Stellar Phenomena (REISEP) at Fukuoka University and an associated project (No. 207002), and JICFuS as "Program for Promoting researches on the Supercomputer Fugaku" (Toward a unified view of the universe: from large scale structures to planets).

\section{References}

[1] Podsiadlowski, P., PASP, 104, 717 (1992)

[2] Urushibata, T., Takahashi, K., Umeda, H., et al., MNRAS, 473, L101 (2018)

[3] Nakamura, K., Takiwaki, T., Kuroda, T., et al., PASJ, 67, 107 (2015)

[4] Nakamura, K., Takiwaki, T., \& Kotake, K., PASJ, 71, 98 (2019)

[5] Kotake, K., Takiwaki, T., Fischer, T., et al., ApJ, 853, 170 (2018) 